\title{
Análise genética do peso em um rebanho de bovinos Nelore
}

\author{
Tiago Luciano Passafaro(1), Breno de Oliveira Fragomeni ${ }^{(2)}$, Daniel Resende Gonçalves ${ }^{(3)}$, \\ Mariana Mamedes de Moraes ${ }^{(1)}$ e Fabio Luiz Buranelo Toral ${ }^{(1)}$
}

\begin{abstract}
(1)Universidade Federal de Minas Gerais, Avenida Antônio Carlos, no 6627, Pampulha, CEP 31270-901 Belo Horizonte, MG, Brasil. E-mail: tpassafaro@hotmail.com, mamedesm@hotmail.com, flbtoral@ufmg.br (2)University of Georgia, River Road, no 425, 30602 Athens, Georgia, EUA. E-mail: brenof@gmail.com ${ }^{(3)}$ Fazenda Mundo Novo, BR-050, Km 125, Caixa Postal 6.006, CEP 38040-970 Uberaba, MG, Brasil. E-mail: drgoncalves@fazendamundonovo.com
\end{abstract}

\begin{abstract}
Resumo - O objetivo deste trabalho foi determinar os parâmetros genéticos para o peso de bovinos Nelore, do nascimento até 1.000 dias de idade, por meio de modelos de regressão aleatória. Utilizaram-se 115.096 registros de peso de 19.417 animais. Os parâmetros genéticos foram obtidos por modelos de regressão aleatória via inferência bayesiana. A trajetória média de crescimento foi ajustada com um polinômio de Legendre quártico. $\mathrm{O}$ efeito genético aditivo direto foi ajustado com um polinômio quadrático de Legendre. Os efeitos de ambiente permanente direto e materno foram ajustados com polinômios de Legendre quíntico e quadrático, respectivamente. A variância residual foi modelada com três classes de idades. Os valores genéticos dos pesos, do nascimento até 1.000 dias de idade, foram utilizados para a análise da tendência genética, por meio de superfícies de resposta. As herdabilidades variaram entre 0,16 e 0,47 . Os efeitos de ambiente permanente direto e materno foram responsáveis por 5 a $77 \%$ e 0,2 a $11 \%$ da variância fenotípica, respectivamente. As correlações genéticas dos pesos em diferentes idades foram altas e superiores a 0,40 . Os valores genéticos foram crescentes ao longo dos anos, e a tendência genética foi máxima para peso aos 500 dias.
\end{abstract}

Termos para indexação: Bos indicus, inferência bayesiana, parâmetro genético, seleção, superfície de resposta.

\section{Genetic analysis of body weight in a Nellore cattle herd}

\begin{abstract}
The objective of this work was to determine the genetic parameters for body weight of Nellore cattle, from birth to 1,000 days of age, by random regression models. Data from 115,096 body weight records of 19,417 animals were used. The genetic parameters were obtained by random regression models and Bayesian inference. The average growth trajectory was fitted by a fourth-order Legendre polynomial. The additive direct effect was fitted by a quadratic Legendre polynomial. Direct and maternal permanent environmental effects were fitted by the fifth- and second-order Legendre polynomials, respectively. Residual variance was modeled by three classes of age. The breeding values of weights obtained from birth to 1,000 days of age were used for the analysis of genetic trend by response surfaces. Heritability ranged from 0.16 to 0.47 . The direct and maternal permanent environmental effects explained 5 to $77 \%$ and 0.2 to $11 \%$ of the phenotypic variance, respectively. The genetic correlations of body weights at different ages were high and above 0.40 . Breeding values increased throughout the years, and genetic trend was maximum for body weight at 500 days.
\end{abstract}

Index terms: Bos indicus, Bayesian inference, genetic parameter, selection, response surface.

\section{Introdução}

O peso é um critério de seleção importante na bovinocultura de corte. Em geral, os pesos padronizados para 120, 205, 365 e 550 dias de idade são analisados com modelos multicaracterísticos. Na edição dos dados para padronização dos pesos, alguns registros obtidos fora de intervalos pré-determinados não são utilizados, o que aumenta o custo com cada registro coletado e efetivamente aproveitado na avaliação genética. Ainda, a avaliação genética multicaracterística de pesos padronizados apresenta menor acurácia do que a avaliação genética com todos os pesos reais disponíveis (Meyer, 2004). Assim, modelos de regressão aleatória (MRA) podem ser mais adequados para a análise genética do crescimento do que os modelos multicaracterísticos. A análise com MRA além de não depender da padronização do peso, permite incluir todos os registros disponíveis (Meyer, 2004) e fornece parâmetros genéticos para todas as idades consideradas (Albuquerque \& Meyer, 2001). 
No Brasil, MRA foram utilizados na análise do crescimento de bovinos Nelore em vários estudos (Albuquerque \& Meyer, 2001; Nobre et al., 2003; Valente et al., 2008; Boligon et al., 2010; Lopes et al., 2012). Trabalhos sobre tendências genéticas de pesos padronizados, com base em resultados de análises multicaracterísticas, também foram realizados quanto a essa raça. Mudanças genéticas positivas ao longo do tempo, para pesos padronizados para 120, 205, 365 e 550 dias, bem como para peso no início da estação de monta, foram relatadas (Mercadante et al., 2003; Souza et al., 2011; Santos et al., 2012). Entretanto, não há estudos de tendência genética quanto ao peso com base em análises de regressão aleatória. Como os MRA permitem obter valores genéticos para o peso em todas as idades, ao longo do intervalo considerado, é possível realizar análise da tendência genética para todas essas idades por meio de superfícies de resposta. O conhecimento dessas tendências genéticas contribuirá para a análise e o planejamento das atividades de programas de melhoramento.

O objetivo deste trabalho foi determinar os parâmetros genéticos para o peso de bovinos Nelore, do nascimento até 1.000 dias de idade, por meio de modelos de regressão aleatória.

\section{Material e Métodos}

Dados de crescimento de bovinos Nelore nascidos entre 1995 e 2012 foram utilizados neste trabalho. O rebanho foi criado em uma fazenda em Brotas, São Paulo ( $22^{\circ} 10^{\prime} 45^{\prime \prime} \mathrm{S}$ e $48^{\circ} 01^{\prime} 21^{\prime \prime} \mathrm{W}$, à altitude de $\left.647 \mathrm{~m}\right)$, com classificação climática Cfa de Köppen-Geiger, até 2000. Em 2001, os animais foram transferidos para outra fazenda, situada em Uberaba, MG (19²4'33"S e $48^{\circ} 06^{\prime} 34^{\prime \prime} \mathrm{W}$, à altitude de $840 \mathrm{~m}$ ), e classificação Aw de Köppen-Geiger. Os animais foram criados em pasto, com suplementação mineral à vontade, durante todo $o$ ano. Os nascimentos concentraram-se nos meses de julho a dezembro.

Pesos do nascimento aos 1.000 dias de idade foram considerados. Descartaram-se os registros superiores ou inferiores a quatro desvios-padrão, em relação à média de cada idade, e os dados dos grupos de contemporâneos (GC) com menos de quatro observações. Os GC foram compostos pela concatenação do ano de nascimento, grupo de manejo, sexo e data da pesagem. Os grupos de manejo na fase de cria, com aproximadamente 30 vacas e seus bezerros, foram formados em ordem sequencial de nascimentos e serviram como referência das condições de criação impostas aos animais. $\mathrm{Na}$ recria, os animais foram reagrupados em novos lotes, mas as informações dos grupos de manejo de cria ficaram concatenadas. Os GC quanto ao peso ao nascimento foram formados por animais nascidos no mesmo ano e do mesmo sexo. $\mathrm{O}$ arquivo final foi composto por 115.096 registros de pesos de 19.417 animais, filhos de 4.879 vacas e 732 touros. Do total de animais, 2.089, 1.590, 3.271, 1.596, $2.467,892,734,983,1.803,1.579,614,625,398,359$, $253,122,33$ e 9 apresentaram 1, 2, 3, 4, 5, 6, 7, 8, 9, 10, $11,12,13,14,15,16,17$ e 18 registros, respectivamente. Dos animais que tiveram progênies $-27,86 \%$ dos touros e $69,14 \%$ das vacas -, também tiveram seus próprios registros de peso na base de dados.

Os dados foram analisados por meio de modelos de regressão aleatória. Os modelos contemplaram os efeitos sistemáticos de GC, idade da vaca ao parto (efeitos linear e quadrático) e a trajetória média de crescimento. Como efeitos aleatórios, consideraram-se os efeitos genético aditivo direto e materno, de ambiente permanente direto e materno e também o resíduo. A trajetória média foi ajustada por polinômios de Legendre que variaram do cúbico ao quíntico, ou por polinômios segmentados lineares com quatro nós, aos 205, 365, 550 e 730 dias. Nessas análises, os polinômios de Legendre do quinto grau foram utilizados para todos os efeitos aleatórios. Após a definição do melhor modelo para o ajuste da trajetória média, as outras análises foram conduzidas para inspecionar a possibilidade de simplificação do modelo, conforme detalhado a seguir.

Polinômios de Legendre desde lineares até os quínticos foram utilizados para ajuste do efeito genético aditivo direto. Nessas análises, os efeitos aditivo materno e de ambiente permanente direto e materno foram ajustados com polinômios do quinto grau. Após a definição do modelo para ajuste do efeito aditivo direto, realizaram-se análises semelhantes para definir a ordem dos polinômios para os efeitos aditivo materno e para os de ambiente permanente direto e materno. Nessas análises, a variância residual foi modelada com 15 classes de idade: $0-14,15-121,122$ 145, 146-195, 196-215, 216-248, 249-320, 321-365, 366-405, 406-459, 460-517, 518-553, 554-596, 597$747,748-1.000$ dias. Os intervalos foram definidos para manter números de dados semelhantes $(>6.000$ observações) em cada classe. Em seguida, verificouse a possibilidade de redução do número de classes. As classes de variância residual adjacentes, em que as 
diferenças dos valores de variâncias foram menores do que $10,20,50,100,150$ e $200 \%$, em relação ao menor valor de variância residual, foram agrupadas, o que resultou em modelos com 10, 8, 6, 5, 4 e 3 classes de variância residual.

O modelo geral para essas análises pode ser descrito na forma matricial:

$$
\mathrm{y}=\mathrm{Xb}+\mathrm{Z}_{1} \mathrm{a}+\mathrm{Z}_{2} \mathrm{~m}+\mathrm{Z}_{3} \mathrm{p}+\mathrm{Z}_{4} \mathrm{c}+\varepsilon
$$

em que: y é o vetor com as observações; $X$ é a matriz de incidência dos efeitos sistemáticos; $b$ é o vetor com a solução dos efeitos sistemáticos; $Z_{1}$, $Z_{2}, Z_{3}$ e $Z_{4}$ são as matrizes de incidência dos efeitos aleatórios genéticos aditivos - direto e materno - e os de ambiente permanente - direto e materno -, respectivamente; $\mathrm{a}, \mathrm{m}, \mathrm{p}$ e c são os vetores com as soluções dos efeitos aleatórios genéticos aditivos direto e materno, de ambiente permanente direto e materno, respectivamente; $\varepsilon$ é o vetor dos resíduos associados a cada observação.

Assumiu-se que,

$\mathrm{y} \mid \mathrm{b}, \mathrm{a}, \mathrm{m}, \mathrm{p}, \mathrm{c}, \sigma_{\mathrm{e}_{1}}^{2} \ldots \sigma_{\mathrm{e}_{\mathrm{x}}}^{2} \sim \mathrm{NMV}\left(\mathrm{Xb}+\mathrm{Z}_{1} \mathrm{a}+\mathrm{Z}_{2} \mathrm{~m}+\mathrm{Z}_{3} \mathrm{p}+\mathrm{Z}_{4} \mathrm{c}, \mathrm{R}\right)$, $\mathrm{R}=\operatorname{diag}\left\{\sigma_{\mathrm{e}_{\mathrm{x}}}^{2}\right\}$,

em que: $x$ representa a classe de variância residual.

As distribuições assumidas a priori foram:

$\mathrm{b} \sim$ constante; $\mathrm{a} \mid \mathrm{K}_{\mathrm{a}} \sim \operatorname{NMV}(0, \mathrm{G})$ com $\mathrm{G}=\mathrm{K}_{\mathrm{a}} \otimes \mathrm{A}$; $\mathrm{m} \mid \mathrm{K}_{\mathrm{m}} \sim \operatorname{NMV}(0, \mathrm{M}) \quad$ com $\mathrm{M}=\mathrm{K}_{\mathrm{m}} \otimes \mathrm{A} ; \quad \mathrm{p} \mid \mathrm{K}_{\mathrm{p}} \sim$ $\operatorname{NMV}(0, \mathrm{P})$ com $\mathrm{P}=\mathrm{K}_{\mathrm{p}} \otimes \mathrm{I}_{\mathrm{p}} ; \mathrm{c} \mid \mathrm{K}_{\mathrm{c}} \sim \operatorname{NMV}(0, \mathrm{C})$ com $\quad \mathrm{C}=\mathrm{K}_{\mathrm{c}} \otimes \mathrm{I}_{\mathrm{c}} ; \quad \mathrm{K}_{\mathrm{a}} \mid \mathrm{v}_{\mathrm{a}}, \mathrm{S}_{\mathrm{a}}^{2} \sim \mathrm{W}^{-1}\left(\mathrm{v}_{\mathrm{a}}, \mathrm{v}_{\mathrm{a}} \mathrm{S}_{\mathrm{a}}^{2}\right)$; $\mathrm{K}_{\mathrm{m}}\left|\mathrm{v}_{\mathrm{m}}, \mathrm{S}_{\mathrm{m}}^{2} \sim \mathrm{W}^{-1}\left(\mathrm{v}_{\mathrm{m}}, \mathrm{v}_{\mathrm{m}} \mathrm{S}_{\mathrm{m}}^{2}\right) ; \mathrm{K}_{\mathrm{p}}\right| \mathrm{v}_{\mathrm{p}}, \mathrm{S}_{\mathrm{p}}^{2} \sim \mathrm{W}^{-1}\left(\mathrm{v}_{\mathrm{p}}, \mathrm{v}_{\mathrm{p}} \mathrm{S}_{\mathrm{p}}^{2}\right)$; $\mathrm{K}_{\mathrm{c}} \mid \mathrm{v}_{\mathrm{c}}, \mathrm{S}_{\mathrm{c}}^{2} \sim \mathrm{W}^{-1}\left(\mathrm{v}_{\mathrm{c}}, \mathrm{v}_{\mathrm{c}} \mathrm{S}_{\mathrm{c}}^{2}\right) ; \quad$ e $\quad \mathrm{R} \mid \mathrm{v}_{\mathrm{e}}, \mathrm{S}_{\mathrm{e}}^{2} \sim \mathrm{W}^{-1}\left(\mathrm{v}_{\mathrm{e}}, \mathrm{v}_{\mathrm{e}} \mathrm{S}_{\mathrm{e}}^{2}\right)$.

Nessas equações, $K_{a}, K_{m}, K_{p}$ e $K_{c}$ representam matrizes de covariância dos coeficientes de regressão dos efeitos genéticos aditivos direto e materno e de ambiente permanente direto e materno, respectivamente; NMV é a distribuição normal multivariada; A é a matriz de parentesco de Wright (23.974 animais); $I_{p}$ e $I_{c}$ representam as matrizes identidade de ordens iguais aos números de animais com dados e de vacas com filhos avaliados, respectivamente; $\mathrm{R}$ representa a matriz de variâncias residuais; $v_{\mathrm{a}}, \mathrm{S}_{\mathrm{a}}^{2}, \mathrm{v}_{\mathrm{m}}, \mathrm{S}_{\mathrm{m}}^{2}, \mathrm{v}_{\mathrm{p}}, \mathrm{S}_{\mathrm{p}}^{2}, \mathrm{v}_{\mathrm{c}}, \mathrm{S}_{\mathrm{c}}^{2}, \mathrm{v}_{\mathrm{e}}$ e $\mathrm{S}_{\mathrm{e}}^{2}$ representam os graus de liberdade e os valores a priori para as covariâncias genéticas aditivas direta e materna, de ambiente permanente direta e materna, e residual, respectivamente; e $\mathrm{W}^{-1}$ é a distribuição de Wishart invertida. Detalhes sobre as distribuições condicionais completas podem ser obtidas em Rekaya et al. (1999).

Amostras das distribuições condicionais completas foram obtidas com o amostrador de Gibbs, por meio do programa GIBBS3F90 (Misztal et al., 2014). Consideraram-se cadeias de 330 mil amostras, com descarte inicial de 30 mil amostras e amostragens a cada 100 ciclos. O tamanho da cadeia foi definido em análises preliminares, conforme Raftery \& Lewis (1992), disponível no programa Bayesian output analysis (BOA) (Smith, 2005), do pacote estatístico $\mathrm{R}$ (R Foundation for Statistical Computing, Viena, Áustria). As convergências das cadeias foram avaliadas pelo critério de Geweke (1992), disponível no mesmo pacote, e por inspeção dos valores amostrados.

Três critérios de ajuste foram calculados - a variância residual total (VRT), o critério de informação da deviance (CID) e o critério de informação bayesiana (CIB) -, descritos de acordo com Pereira et al. (2013). Em seguida, construiu-se um índice pela soma desses três critérios, conforme sugestão de Liu et al. (2006). Os menores valores do índice indicam os modelos de melhor ajuste aos dados.

Calcularam-se os valores genéticos quanto ao peso do nascimento aos 1.000 dias, a partir das médias a posteriori dos coeficientes de regressão aditivo direto de cada animal com alguma pesagem válida. Esses valores também foram expressos em unidades de desvios-padrão (UDP) genéticos, dividindo-se os valores genéticos pela média, a posteriori, do desvio-padrão genético em cada idade. Os valores genéticos, expressos em quilogramas e padronizados em UDP, foram utilizados para estudo da tendência genética por meio de superfície de resposta. Utilizouse o seguinte modelo de superfície de resposta,

$$
\mathrm{VG}_{\mathrm{jp}}=\mathrm{b}_{0}+\mathrm{b}_{1} \mathrm{id}_{\mathrm{j}}+\mathrm{b}_{2} \mathrm{id}_{\mathrm{j}}^{2}+\mathrm{b}_{3} \mathrm{~s}_{\mathrm{p}}+\mathrm{b}_{4} \mathrm{~s}_{\mathrm{p}}^{2}+\mathrm{b}_{13} \mathrm{id}_{\mathrm{j}} \mathrm{s}_{\mathrm{p}}+\varepsilon_{\mathrm{jp}}
$$

em que: $V_{\mathrm{jp}}$ são os valores genéticos (kg ou UDP), para peso na idade $\mathrm{j}(\mathrm{j}=0, \ldots, 1.000)$ dos animais nascidos na safra $p(p=0, \ldots, 17) ; b_{0}$ é o intercepto; $b_{1}$ e $b_{2}$ são os coeficientes de regressão linear e quadrático para $a$ idade, respectivamente; $b_{3}$ e $b_{4}$ são respectivamente coeficientes de regressão linear e quadrático para a safra; $b_{12}$ é o coeficiente de regressão da interação entre safra e idade; $\mathrm{S}_{\mathrm{p}}$ safra $\mathrm{p}, \mathrm{id}_{\mathrm{j}}$ é a idade $\mathrm{j}$, e $\varepsilon_{\mathrm{jp}}$ é o resíduo. A análise de superfície de resposta foi realizada pelo programa R (R Foundation for Statistical Computing, Viena, Áustria). 


\section{Resultados e Discussão}

O polinômio de Legendre de quarta ordem ajustouse melhor à trajetória média de crescimento, para os dados de pesos de bovinos Nelore, em comparação aos polinômios segmentado e de Legendre do terceiro e quinto graus (Tabela 1). Tal resultado corrobora aqueles obtidos por Boligon et al. (2010), que analisaram o crescimento de bovinos Nelore do nascimento à idade adulta. $\mathrm{O}$ ajuste da trajetória de crescimento do

Tabela 1. Número total de componentes de covariância $(\mathrm{NCV})$ e critérios $^{(1)}$ para comparação de modelos de regressão aleatória para análise do peso de bovinos Nelore do nascimento aos 1.000 dias de idade.

\begin{tabular}{|c|c|c|c|c|c|}
\hline Modelo $^{(2)}$ & $\mathrm{NCV}$ & VRT & CID & CIB & Índice \\
\hline & \multicolumn{5}{|c|}{ Modelo de referência } \\
\hline \multirow[t]{2}{*}{$\underline{\mathrm{L}(6) \mathrm{L}(6666) \mathrm{R}(15)}$} & 99 & $71.552,20$ & $785.937,27$ & $742.469,09$ & $1.599 .958,56$ \\
\hline & \multicolumn{5}{|c|}{ Trajetória média de crescimento } \\
\hline $\mathrm{L}(5) \mathrm{L}(6666) \mathrm{R}(15)$ & 99 & $71.471,22$ & $785.214,17$ & $742.273,88$ & $1.598 .959,27$ \\
\hline $\mathrm{L}(4) \mathrm{L}(6666) \mathrm{R}(15)$ & 99 & $71.618,71$ & $785.550,55$ & $742.289,42$ & $1.599 .458,68$ \\
\hline \multirow[t]{2}{*}{$\mathrm{S}(6) \mathrm{L}(6666) \mathrm{R}(15)$} & 99 & $71.473,97$ & $785.336,39$ & $742.649,09$ & $1.599 .459,45$ \\
\hline & \multicolumn{5}{|c|}{ Funções de covariância para o efeito genético aditivo direto } \\
\hline $\mathrm{L}(5) \mathrm{L}(6666) \mathrm{R}(15)$ & 99 & $71.471,22$ & $785.214,17$ & $742.273,88$ & $1.598 .959,27$ \\
\hline $\mathrm{L}(5) \mathrm{L}(5666) \mathrm{R}(15)$ & 93 & $71.423,90$ & $786.227,79$ & $742.290,94$ & $1.599 .942,63$ \\
\hline $\mathrm{L}(5) \mathrm{L}(4666) \mathrm{R}(15)$ & 88 & $71.387,91$ & $786.659,13$ & $742.338,54$ & $1.600 .385,58$ \\
\hline $\mathrm{L}(5) \mathrm{L}(3666) \mathrm{R}(15)$ & 84 & $71.407,75$ & $785.475,65$ & $741.623,82$ & $1.598 .507,22$ \\
\hline \multirow[t]{2}{*}{$\mathrm{L}(5) \mathrm{L}(2666) \mathrm{R}(15)$} & 81 & $71.711,10$ & $786.139,00$ & $741.475,48$ & $1.599 .325,58$ \\
\hline & \multicolumn{5}{|c|}{ Funções de covariância para o efeito genético aditivo materno } \\
\hline $\mathrm{L}(5) \mathrm{L}(3666) \mathrm{R}(15)$ & 84 & $71.407,75$ & $785.475,65$ & $741.623,82$ & $1.598 .507,22$ \\
\hline $\mathrm{L}(5) \mathrm{L}(3566) \mathrm{R}(15)$ & 78 & $71.678,76$ & $786.258,30$ & $740.582,99$ & $1.598 .520,06$ \\
\hline $\mathrm{L}(5) \mathrm{L}(3466) \mathrm{R}(15)$ & 73 & $71.335,75$ & $786.241,13$ & $742.267,60$ & $1.599 .844,48$ \\
\hline $\mathrm{L}(5) \mathrm{L}(3366) \mathrm{R}(15)$ & 69 & $71.446,17$ & $786.246,30$ & $741.578,93$ & $1.599 .271,41$ \\
\hline $\mathrm{L}(5) \mathrm{L}(3266) \mathrm{R}(15)$ & 66 & $71.663,47$ & $786.334,89$ & $741.386,27$ & $1.599 .384,63$ \\
\hline \multirow[t]{2}{*}{$\mathrm{L}(5) \mathrm{L}(3066) \mathrm{R}(15)$} & 63 & $71.541,65$ & $785.432,71$ & $740.795,99$ & $1.597 .770,34$ \\
\hline & \multicolumn{5}{|c|}{ Funções de covariância para o efeito de ambiente permanente direto } \\
\hline $\mathrm{L}(5) \mathrm{L}(3066) \mathrm{R}(15)$ & 63 & $71.541,65$ & $785.432,71$ & $740.795,99$ & $1.597 .770,34$ \\
\hline $\mathrm{L}(5) \mathrm{L}(3056) \mathrm{R}(15)$ & 57 & $74.812,77$ & $788.710,93$ & $744.550,60$ & $1.608 .074,30$ \\
\hline $\mathrm{L}(5) \mathrm{L}(3046) \mathrm{R}(15)$ & 52 & $80.098,91$ & $784.433,66$ & $740.103,87$ & $1.604 .636,44$ \\
\hline $\mathrm{L}(5) \mathrm{L}(3036) \mathrm{R}(15)$ & 48 & $87.453,12$ & $794.661,73$ & $750.684,60$ & $1.632 .799,45$ \\
\hline \multirow[t]{2}{*}{$\mathrm{L}(5) \mathrm{L}(3026) \mathrm{R}(15)$} & 45 & $87.968,82$ & $796.306,38$ & $752.619,60$ & $1.636 .894,81$ \\
\hline & \multicolumn{5}{|c|}{ Funções de covariâncias para o efeito de ambiente permanente materno } \\
\hline $\mathrm{L}(5) \mathrm{L}(3066) \mathrm{R}(15)$ & 63 & $71.541,65$ & $785.432,71$ & $740.795,99$ & $1.597 .770,34$ \\
\hline $\mathrm{L}(5) \mathrm{L}(3065) \mathrm{R}(15)$ & 57 & $71.400,80$ & $786.750,72$ & $741.537,67$ & $1.599 .689,18$ \\
\hline L(5)L(3064)R(15) & 52 & $71.493,78$ & $786.097,71$ & $740.525,14$ & $1.598 .116,63$ \\
\hline $\mathrm{L}(5) \mathrm{L}(3063) \mathrm{R}(15)$ & 48 & $71.491,29$ & $785.487,09$ & $740.727,47$ & $1.597 .705,85$ \\
\hline $\mathrm{L}(5) \mathrm{L}(3062) \mathrm{R}(15)$ & 45 & $71.440,47$ & $786.570,49$ & $741.248,69$ & $1.599 .259,66$ \\
\hline \multirow[t]{2}{*}{$\mathrm{L}(5) \mathrm{L}(3060) \mathrm{R}(15)$} & 42 & $71.392,54$ & $786.656,81$ & $740.820,88$ & $1.598 .870,22$ \\
\hline & \multicolumn{5}{|c|}{ Variância residual } \\
\hline $\mathrm{L}(5) \mathrm{L}(3063) \mathrm{R}(15)$ & 48 & $71.491,29$ & $785.487,09$ & $740.727,47$ & $1.597 .705,85$ \\
\hline $\mathrm{L}(5) \mathrm{L}(3063) \mathrm{R}(10)$ & 43 & $70.407,60$ & $785.338,56$ & $740.688,80$ & $1.596 .434,96$ \\
\hline $\mathrm{L}(5) \mathrm{L}(3063) \mathrm{R}(08)$ & 41 & $70.759,68$ & $786.232,65$ & $740.627,03$ & $1.597 .619,36$ \\
\hline $\mathrm{L}(5) \mathrm{L}(3063) \mathrm{R}(06)$ & 39 & $70.556,39$ & $786.890,41$ & $740.951,87$ & $1.598 .398,67$ \\
\hline $\mathrm{L}(5) \mathrm{L}(3063) \mathrm{R}(05)$ & 38 & $70.940,80$ & $787.042,21$ & $740.807,23$ & $1.598 .790,24$ \\
\hline $\mathrm{L}(5) \mathrm{L}(3063) \mathrm{R}(04)$ & 37 & $64.601,59$ & $786.620,24$ & $741.251,75$ & $1.592 .473,58$ \\
\hline $\mathrm{L}(5) \mathrm{L}(3063) \mathrm{R}(03)$ & 36 & $63.436,36$ & $783.980,39$ & $739.181,00$ & $1.586 .597,75$ \\
\hline $\mathrm{L}(5) \mathrm{L}(3063) \mathrm{R}(01)$ & 34 & $39.689,17$ & $796.359,50$ & $755.268,12$ & $1.591 .316,80$ \\
\hline
\end{tabular}

(1)VRT, variância residual total; CID, critério de informação da deviance; CIB, critério de informação bayesiana; Índice, VRT + CID + CIB. (2)X(k $\left.\mathrm{k}_{\mathrm{tm}}\right)$ $\mathrm{L}\left(\mathrm{k}_{\mathrm{a}} \mathrm{k}_{\mathrm{m}} \mathrm{k}_{\mathrm{p}} \mathrm{k}_{\mathrm{c}}\right) \mathrm{R}(\mathrm{r})$, $\mathrm{X}$, tipo de polinômio $\left(\mathrm{L}=\right.$ Legendre, $\mathrm{S}=$ segmentado); $\mathrm{k}_{\mathrm{tm}}, \mathrm{k}_{\mathrm{a}}, \mathrm{k}_{\mathrm{m}}, \mathrm{k}_{\mathrm{p}}, \mathrm{k}_{\mathrm{c}}$, coeficientes de regressão da trajetória média, efeitos genéticos aditivos direto e materno, e efeitos de ambiente permanente direto e materno, respectivamente; $\mathrm{R}(\mathrm{r})$, número de classes de variância residual. 
nascimento aos dois anos de idade, em bovinos Nelore, tem sido realizado por meio de polinômios de Legendre do terceiro grau (Albuquerque \& Meyer, 2001; Nobre et al., 2003; Lopes et al., 2012). A necessidade de maior ordem para ajuste pode ser atribuída às oscilações das médias de peso, ao longo do maior intervalo de idades (Figura 1).

Mudanças na ordem do polinômio de Legendre, para o efeito aditivo direto, resultaram na escolha do polinômio quadrático (Tabela 1, Figura 2 A). Esse resultado corrobora os de Meyer (2005), Valente et al. (2008) e Toral et al. (2014). No entanto, Albuquerque $\&$ Meyer (2001), Dias et al. (2006), Boligon et al. (2010) e Lopes et al. (2012) utilizaram polinômios cúbicos, e Mota et al. (2013) utilizaram polinômios quínticos para ajuste do mesmo efeito. Apesar dessas discrepâncias, a análise da razão entre o maior e o menor autovalor (Tabela 2) mostrou que este último é muito pequeno, o que indica que o polinômio do segundo grau é o adequado.

A redução do número de coeficientes de regressão para o efeito de ambiente permanente direto (Figura 2 B) resultou em aumento do valor do índice (Tabela 1). Isto está de acordo com Albuquerque \& Meyer (2001), Dias et al. (2006) e Boligon et al. (2010). Os autores citados relataram a necessidade de modelar o efeito de ambiente permanente direto com polinômios de Legendre de ordem elevada. $\mathrm{O}$ ajuste desse efeito, com

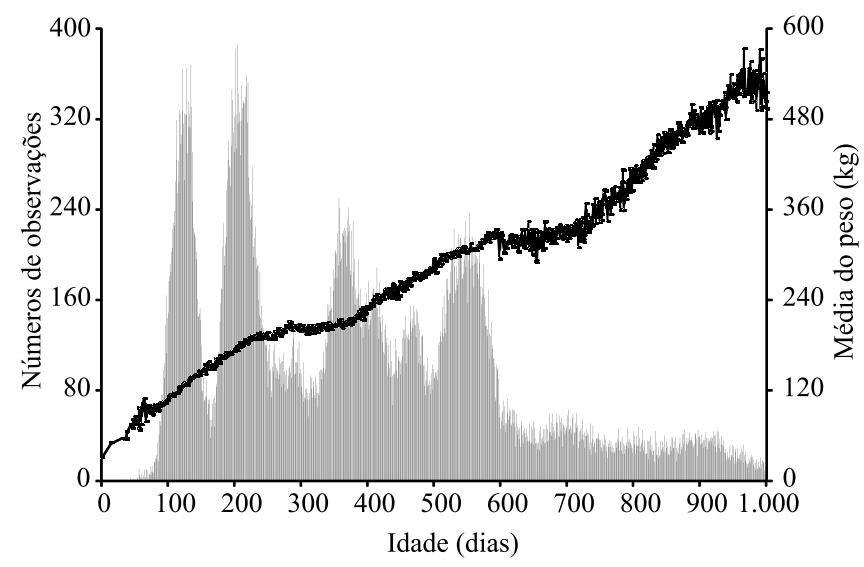

Figura 1. Número de observações (barras em cinza, eixo à esquerda) e média (linha em preto, eixo à direita) de peso de bovinos Nelore do nascimento aos 1.000 dias de idade. O número de registros de peso ao nascimento (19.291) foi omitido na figura. polinômios de maior grau, está relacionado à dificuldade de sua separação do efeito genético aditivo direto e por problemas de amostragens (Meyer, 2001). Além disso, a variação do número de observações, em cada idade, e as diferenças entre o intervalo de mensurações, para cada animal, também podem dificultar a separação dos efeitos diretos (Toral et al., 2014).

Alterações na ordem do polinômio de Legendre, para os efeitos genético e de ambiente permanente maternos, resultaram na indicação do modelo que incluiu apenas o efeito de ambiente permanente materno, ajustado com um polinômio quadrático (Tabela 1, Figura 2 C), como adequado. A separação dos efeitos genético materno e de ambiente materno permanente é complexa (Meyer, 1992) e, assim como no presente trabalho, outros autores (Albuquerque \& Meyer, 2001; Cyrillo et al., 2004; Mota et al., 2013) também incluíram apenas um efeito materno na análise genética do peso de bovinos de corte. Albuquerque \& Meyer (2001) analisaram três bases de dados de pesos de bovinos Nelore, do nascimento aos 630 dias de idade, e verificaram que a inclusão de um único efeito materno (o efeito de ambiente permanente) foi suficiente para a análise genética em duas de suas bases de dados. Resultado semelhante foi obtido por Cyrillo et al. (2004), que analisaram o peso de bovinos Nelore do nascimento aos 378 dias de idade. Mota et al. (2013) optaram pela inclusão do efeito genético materno, ao invés do efeito de ambiente materno permanente, para a análise do peso de bovinos Simbrasil, do nascimento aos 810 dias de idade.

A semelhança entre os valores de variância residual permitiu o agrupamento de classes (Figura 2 D). $\mathrm{O}$ modelo com três classes apresentou os menores valores para o índice de classificação de modelos (Tabela 1). Essa abordagem permitiu a identificação de um modelo parcimonioso, sem comprometer a qualidade da análise. $\mathrm{O}$ ajuste da variância residual para dados de peso de zebuínos, com três classes de variância, também foi realizado por Albuquerque \& Meyer (2001) e Toral et al. (2014). A seleção do número de classes de variância definida pelo índice apresentou alguns aspectos que devem ser avaliados com cautela. A VRT foi menor para o modelo com variância residual homogênea, e o CID e o BIC do mesmo modelo foram maiores em comparação aos modelos ajustados com variância residual heterogênea (Tabela 1). Se a escolha do modelo fosse 
baseada apenas na VRT o modelo de melhor ajuste seria o que considera a variância residual homogênea, resultado que seria incoerente com as expectativas.
Os efeitos de ambiente temporário, em bovinos de corte, não são constantes ao longo do tempo (Dias et al., 2006; Scalez et al., 2014; Toral et al., 2014).
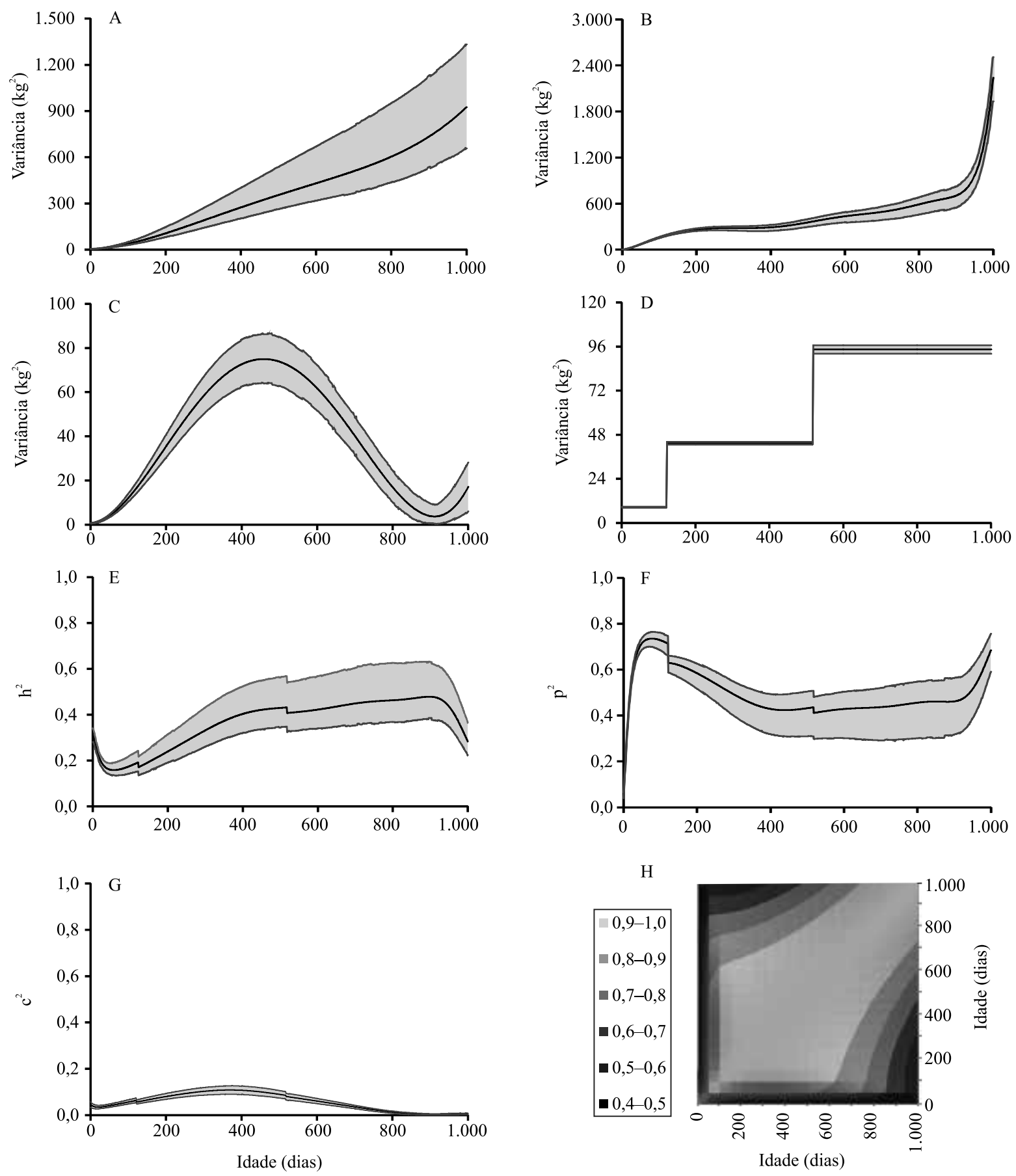

Figura 2. Médias a posteriori e limites dos intervalos de alta densidade ( $95 \%$ das amostras) de parâmetros genéticos, quanto ao peso de bovinos Nelore, do nascimento aos 1.000 dias de idade: A, variância genética aditiva direta; B, variância de ambiente permanente direto; $\mathrm{C}$, variância de ambiente permanente materno; $\mathrm{D}$, variância residual; $\mathrm{E}$, herdabilidade $\left(\mathrm{h}^{2}\right) ; \mathrm{F}$, razão da variância de ambiente permanente direto em relação à variância fenotípica $\left(p^{2}\right)$; $G$, razão da variância de ambiente permanente materna $\left(\mathrm{c}^{2}\right)$ em relação à variância fenotípica, e correlações genéticas $(\mathrm{H})$. 
A redução no número de observações ao longo do tempo, as dificuldades para ajuste da trajetória média e a existência de tratamentos diferenciados - e não considerados no modelo estatístico, pelo fato de não estarem registrados no arquivo zootécnico - podem contribuir para a existência de variâncias residuais heterogêneas. Assim, a VRT deve ser avaliada em conjunto com outros critérios de seleção de modelos.

Tabela 2. Médias a posteriori e limites dos intervalos de alta densidade (com 95\% das amostras) da variância (diagonal), covariância (abaixo da diagonal), e correlações (acima da diagonal) dos coeficientes de regressão, associados aos efeitos genético aditivo direto e aos efeitos de ambiente permanente direto e materno, e autovalores $(\lambda)$ das matrizes de covariância, para a análise do peso de bovinos Nelore do nascimento aos 1.000 dias de idade.

\begin{tabular}{|c|c|c|c|c|c|c|c|}
\hline Ordem & 0 & 1 & 2 & 3 & 4 & 5 & $\lambda$ \\
\hline & \multicolumn{7}{|c|}{ Efeito aditivo direto (média) } \\
\hline 0 & 281,10 & 0,84 & $-0,37$ & - & - & - & 460,56 \\
\hline 1 & 206,33 & 216,76 & 0,19 & - & - & - & 112,31 \\
\hline \multirow[t]{2}{*}{2} & $-54,62$ & 23,77 & 76,05 & - & - & - & 1,03 \\
\hline & \multicolumn{7}{|c|}{ Efeito aditivo direto (limite inferior) } \\
\hline 0 & 207,20 & 0,77 & $-0,51$ & - & - & - & 333,36 \\
\hline 1 & 144,00 & 155,20 & 0,05 & - & - & - & 84,81 \\
\hline \multirow[t]{2}{*}{2} & $-87,06$ & $-8,87$ & 76,05 & - & - & - & 0,83 \\
\hline & \multicolumn{7}{|c|}{ Efeito aditivo direto (limite superior) } \\
\hline 0 & 438,20 & 0,90 & $-0,23$ & - & - & - & 727,08 \\
\hline 1 & 337,30 & 320,60 & 0,39 & - & - & - & 144,10 \\
\hline \multirow[t]{2}{*}{2} & $-29,22$ & 50,62 & 76,05 & - & - & - & 1,24 \\
\hline & \multicolumn{7}{|c|}{ Efeito permanente direto (média) } \\
\hline 0 & 297,41 & 0,70 & $-0,13$ & 0,26 & $-0,07$ & 0,18 & 447,16 \\
\hline 1 & 168,08 & 191,68 & 0,18 & 0,06 & 0,22 & 0,25 & 297,29 \\
\hline 2 & $-22,39$ & 25,27 & 99,63 & 0,47 & 0,14 & 0,14 & 144,12 \\
\hline 3 & 52,92 & 10,33 & 54,78 & 136,75 & 0,38 & 0,19 & 99,81 \\
\hline 4 & $-16,92$ & 43,92 & 20,12 & 65,08 & 210,54 & 0,77 & 39,40 \\
\hline \multirow[t]{2}{*}{5} & 29,46 & 33,52 & 13,34 & 21,36 & 106,67 & 91,76 & 0,00 \\
\hline & \multicolumn{7}{|c|}{ Efeito permanente direto (limite inferior) } \\
\hline 0 & 241,80 & 0,62 & $-0,23$ & 0,18 & $-0,12$ & 0,12 & 368,67 \\
\hline 1 & 122,10 & 153,40 & 0,06 & $-0,01$ & 0,14 & 0,17 & 258,67 \\
\hline 2 & $-38,97$ & 7,14 & 82,31 & 0,40 & 0,05 & 0,04 & 130,75 \\
\hline 3 & 39,13 & $-1,85$ & 43,62 & 123,00 & 0,32 & 0,11 & 83,17 \\
\hline 4 & $-31,65$ & 30,14 & 6,70 & 50,40 & 189,90 & 0,74 & 36,77 \\
\hline \multirow[t]{2}{*}{5} & 20,50 & 24,77 & 4,22 & 12,75 & 93,96 & 83,76 & $-0,03$ \\
\hline & \multicolumn{7}{|c|}{ Efeito permanente direto (limite superior) } \\
\hline 0 & 335,90 & 0,76 & $-0,03$ & 0,34 & 0,00 & 0,24 & 507,26 \\
\hline 1 & 206,10 & 231,30 & 0,31 & 0,14 & 0,29 & 0,32 & 337,54 \\
\hline 2 & $-3,44$ & 42,96 & 116,10 & 0,53 & 0,22 & 0,23 & 157,42 \\
\hline 3 & 69,06 & 22,89 & 66,38 & 152,50 & 0,44 & 0,26 & 118,24 \\
\hline 4 & $-0,49$ & 58,47 & 33,90 & 80,95 & 231,70 & 0,79 & 42,03 \\
\hline \multirow[t]{2}{*}{5} & 39,43 & 43,23 & 22,70 & 31,09 & 117,40 & 99,35 & 0,03 \\
\hline & \multicolumn{7}{|c|}{ Efeito permanente materno (média) } \\
\hline 0 & 26,99 & $-0,69$ & $-0,99$ & - & - & - & 76,53 \\
\hline 1 & $-8,22$ & 5,19 & 0,79 & - & - & - & 2,45 \\
\hline \multirow[t]{2}{*}{2} & $-35,09$ & 12,31 & 46,81 & - & - & - & 0,02 \\
\hline & \multicolumn{7}{|c|}{ Efeito permanente materno (limite inferior) } \\
\hline 0 & 21,81 & $-0,97$ & $-1,00$ & - & - & - & 65,26 \\
\hline 1 & $-11,08$ & 2,30 & 0,63 & - & - & - & 0,23 \\
\hline \multirow[t]{2}{*}{2} & $-40,55$ & 7,59 & 39,36 & - & - & - & 0,00 \\
\hline & \multicolumn{7}{|c|}{ Efeito permanente materno (limite superior) } \\
\hline 0 & 32,07 & $-0,41$ & $-0,95$ & - & - & - & 87,59 \\
\hline 1 & $-4,69$ & 7,05 & 0,98 & - & - & - & 5,01 \\
\hline 2 & $-29,82$ & 17,24 & 54,07 & - & - & - & 0,18 \\
\hline
\end{tabular}


As médias a posteriori dos valores de herdabilidade, quanto ao peso de bovinos Nelore, variaram de 0,16 - aos 56 dias -, a 0,47 - aos 898 dias (Figura 2 E). Os maiores valores de herdabilidade situaram-se entre 390 e 965 dias, e foram valores iguais ou superiores a 0,40 (Figura 2 E). Esses resultados mostram que a herdabilidade quanto ao peso, em bovinos Nelore, foi de média a alta magnitude, e indicam que a seleção para aumentar o peso pode resultar no incremento da média dessa característica. Além disso, a resposta da seleção será maior no intervalo de 390 a 965 dias, uma vez que, nessa faixa de idade, estão contidos os maiores valores de variância genética aditiva direta (Figura 2 A) e herdabilidade (Figura 2 E).

$\mathrm{O}$ efeito de ambiente permanente direto foi responsável por 5\% (ao nascimento) a 74\% (aos 77 dias) da variação total e, em média, explicou 50\% das variações fenotípicas (Figura $2 \mathrm{~F}$ ). Este resultado mostra que tal efeito foi de alta magnitude (exceto ao nascimento) e tem grande impacto na partição da variância fenotípica total em cada idade. Esses resultados encontram-se próximos dos valores de 55\% de variância fenotípica calculados em outros trabalhos sobre bovinos Nelore (Boligon et al., 2010; Lopes et al., 2012).

$\mathrm{O}$ efeito de ambiente permanente materno foi responsável por explicar de 0,2\% (915 dias) a 11\% (370 dias) da variância fenotípica total, com média de $5 \%$, e maiores valores próximos aos 365 dias de idade
(Figura 2 G). Em geral, estes resultados indicam que o efeito de ambiente permanente materno foi de baixa magnitude, mas respaldam sua inclusão na análise do peso. A exclusão dos efeitos maternos pode implicar na superestimação dos valores de herdabilidade (Nobre et al., 2003) e, posteriormente, ocasionar alterações dos valores genéticos preditos. Os resultados encontrados no presente trabalho são semelhantes aos observados por Albuquerque \& Meyer (2001), Dias et al. (2006) e Boligon et al. (2010).

As médias a posteriori das correlações genéticas dos pesos em diferentes idades foram positivas e de média a alta magnitude, durante todo o intervalo avaliado (Figura $2 \mathrm{H}$ ). Em geral, as correlações foram maiores em idades adjacentes, e menores à medida que a distância entre as idades tornaram-se maiores (Figura 2 H). Este resultado é uma indicação de que a seleção para aumentar o peso, em qualquer idade, resultaria em respostas correlacionadas de mesmo sentido para todas as idades consideradas no intervalo. Resultados semelhantes foram obtidos por Albuquerque \& Meyer (2001) e Boligon et al. (2010).

Todos os coeficientes de regressão das superfícies de resposta das tendências genéticas foram significativos $(p \leq 0,05)$. Os valores genéticos quanto ao peso aumentaram ao longo do tempo (safra), mas as magnitudes das mudanças variaram de acordo com a idade (Figura 3). As maiores mudanças ( $\mathrm{kg}$ ) ocorreram quanto aos pesos nas idades mais avançadas, e as
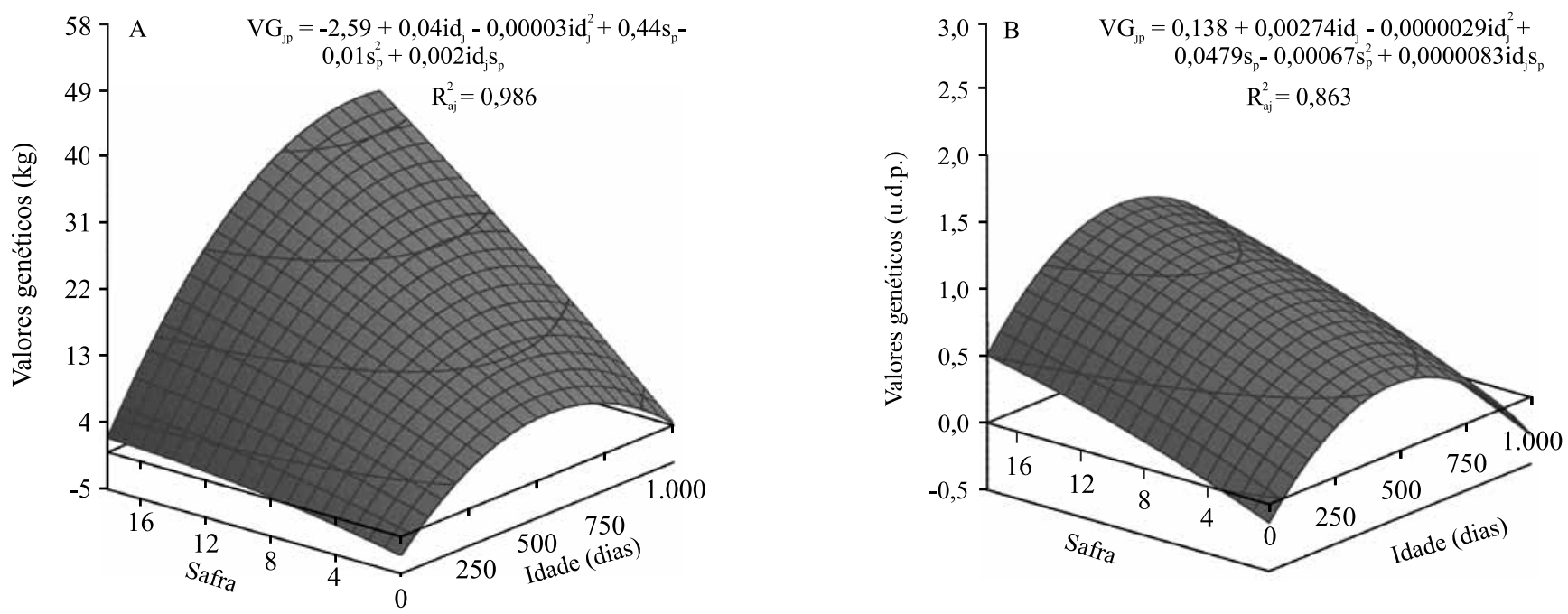

Figura 3. Superfície de resposta dos valores genéticos com base no peso (A) e em unidades de desvios-padrão (B, UDP) do peso de bovinos Nelore do nascimento aos 1.000 dias de idade, em função da safra de nascimento (18 safras).

Pesq. agropec. bras., Brasília, v.51, n.2, p.149-158, fev. 2016

DOI: 10.1590/S0100-204X2016000200007 
menores ocorreram quanto ao peso ao nascimento (Figura $3 \mathrm{~A}$ ). Isso pode ser explicado pelos aumentos das médias (Figura 1) e variâncias (Figura 2) do peso ao longo do tempo. Apesar das correlações positivas do peso ao nascimento com os pesos nas idades mais avançadas, a tendência genética para aquele peso foi próxima de zero porque, no rebanho estudado, a utilização de touros com peso ao nascimento acima de $40 \mathrm{~kg}$ é evitada.

As maiores mudanças (em UDP) foram observadas para os pesos entre 400 e 600 dias, com valor máximo para peso aos 500 dias (Figura $3 \mathrm{~B}$ ). Nesse rebanho, o peso aos 550 dias e o ganho médio diário pós-desmama são as principais características de crescimento consideradas na seleção. Assim, as maiores mudanças (UDP) para o peso próximo dessa idade são reflexos da eficiência do processo de seleção. No rebanho estudado, há descarte de fêmeas jovens, com idade ao primeiro parto superior a 40 meses, e de vacas adultas que não tiveram pelo menos dois partos nos últimos três anos da vida reprodutiva. Como há correlação genética negativa do peso ao primeiro parto (Mello et al., 2014) e do peso adulto (Mello et al., 2006) com o número de bezerros desmamados, durante a vida produtiva de vacas de corte, é possível inferir que o descarte de vacas com baixo desempenho reprodutivo pode ter levado à eliminação de vacas muito pesadas e que tiveram o desempenho reprodutivo prejudicado, por não terem suas exigências nutricionais atendidas no sistema de produção em pasto. Este tipo de estratégia pode ter sido responsável pela redução das tendências genéticas (UDP), para os pesos em idades acima de 600 dias.

Os resultados da literatura também mostram a possibilidade de mudanças genéticas anuais em torno de $1 \mathrm{~kg}$, ou até superiores, para o peso aos 550 dias na raça Nelore (Souza et al., 2011; Santos et al., 2012). Diferenças entre os trabalhos podem ser explicadas por diferenças de estruturas populacionais (rebanho fechado x rebanho aberto), dos critérios de seleção e das bases genéticas.

A seleção de animais mais pesados ao sobreano, com pesos moderados ao nascimento, e o descarte de vacas de baixo desempenho reprodutivo podem promover mudanças genéticas quanto ao peso na idade de interesse (sobreano), sem prejudicar o peso ao nascimento ou aumentar o peso em idades mais avançadas, acima do suportado pelo sistema de produção em pasto.

\section{Conclusões}

1. Há variabilidade genética suficiente, para promover mudanças nos pesos de bovinos Nelore do nascimento aos 1.000 dias de idade.

2. A herdabilidade quanto ao peso em bovinos Nelore é de média a alta magnitude, o que torna possível a seleção para aumentar o peso; e a resposta da seleção é maior no intervalo de 390 a 965 dias, faixa de idade em que se encontram os maiores valores de variância genética aditiva direta e herdabilidade.

3. A seleção quanto ao peso, em qualquer idade do intervalo, resulta em respostas diretas e correlacionadas quanto ao peso em outras idades.

\section{Agradecimentos}

À Coordenação de Aperfeiçoamento de Pessoal de Nível Superior (Capes), ao Conselho Nacional de Desenvolvimento Científico e Tecnológico (CNPq), e à Fundação de Amparo a Pesquisa do Estado de Minas Gerais (Fapemig), pelo apoio financeiro; ao Eng ${ }^{\circ}$. Agr. Eduardo Penteado Cardoso (Fazenda Mundo Novo), por disponibilizar o banco de dados; e ao Prof. Ignacy Misztal e seus colaboradores, pela disponibilização do programa GIBBS3F90.

\section{Referências}

ALBUQUERQUE, L.G.; MEYER, K. Estimates of covariance functions for growth from birth to 630 days of age in Nelore cattle. Journal of Animal Science, v.79, p.2776-2789, 2001.

BOLIGON, A.A.; MERCADANTE, M.E.Z.; FORNI, S.; LÔBO, R.B.; ALBUQUERQUE, L.G. Covariance functions for weights from birth to maturity in Nellore cows. Journal of Animal Science, v.88, p.849-859, 2010. DOI: 10.2527/jas.2008-1511.

CYRILLO, J.N. dos S.G.; ALENCAR, M.M. de; RAZOOK, A.G.; MERCADANTE, M.E.Z.; FIGUEIREDO, L.A. de. Modelagem e estimação de parâmetros genéticos e fenotípicos para pesos do nascimento à seleção (378 dias) de machos Nelore. Revista Brasileira de Zootecnia, v.33, p.1405-14015, 2004. DOI: 10.1590/ S1516-35982004000600007.

DIAS, L.T.; ALBUQUERQUE, L.G. de; TONHATI, H.; TEIXEIRA, R. de A. Estimação de parâmetros genéticos para peso do nascimento aos 550 dias de idade para animais da raça Tabapuã utilizando-se modelos de regressão aleatória. Revista Brasileira de Zootecnia, v.35, p.1915-1925, 2006. DOI: 10.1590/ S1516-35982006000700006.

GEWEKE, J. Evaluating the accuracy of sampling-based approaches to the calculation of posterior moments. In: BERNARDO, J.M.; BERGER, J.O.; DAWID, A.P.; SMITH, A.F.M. (Ed.). Bayesian statistics 4. Oxford: Oxford University Press, 1992. p.169-193. 
LIU, X.Y.;ZHANG, J.; SCHAEFFER, L.R.; YANG, R.Q.;ZHANG, W.L. Optimal random regression models for milk production in dairy cattle. Journal of Dairy Science, v.89, p.2233-2235, 2006. DOI: $10.3168 /$ jds.S0022-0302(06)72294-9.

LOPES, F.B.; MAGNABOSCO, C.U.; PAULINI, F.; SILVA, M.C. da; MIYAGI, E.S.; LÔBO, R.B. Analysis of longitudinal data of Nellore cattle from performance test at pasture using random regression model. SpringerPlus, v.1, article 49, 2012. DOI: 10.1186/2193-1801-1-49.

MELLO, S. de P.; ALENCAR, M.M. de; TORAL, F.L.B.; GIANLORENÇO, V.K. Estimativas de parâmetros genéticos para características de crescimento e produtividade em vacas da raça Canchim, utilizando-se inferência bayesiana. Revista Brasileira de Zootecnia, v.35, p.92-97, 2006. DOI: 10.1590/ S1516-35982006000100011.

MELLO, S.P.; ALENCAR, M.M.; SANTOS, D.C.C.; TORAL, F.L.B. Análise genética de características de fertilidade, de crescimento e de produtividade em vacas da raça Canchim. Arquivo Brasileiro de Medicina Veterinária e Zootecnia, v.66, p.555-562, 2014. DOI: 10.1590/1678-41626876.

MERCADANTE, M.E.; PACKER, I.U.; RAZOOK, A.G.; CYRILLO, J.N.; FIGUEIREDO, L.A. Direct and correlated responses to selection for yearling weight on reproductive performance of Nelore cows. Journal of Animal Science, v.81, p.376-384, 2003.

MEYER, K. Bias and sampling covariances of estimates of variance components due to maternal effects. Genetics Selection Evolution, v.24, p.487-509, 1992. DOI: 10.1186/1297-9686-24-6-487.

MEYER, K. Estimates of direct and maternal covariance functions for growth of Australian beef calves from birth to weaning. Genetics Selection Evolution, v.33, p.487-514, 2001. DOI: 10.1186/1297-9686-33-5-487.

MEYER, K. Estimates of genetic covariance functions for growth of Angus cattle. Journal of Animal Breeding and Genetics, v.122, p.73-85, 2005. DOI: 10.1111/j.1439-0388.2005.00503.x.

MEYER, K. Scope for a random regression model in genetic evaluation of beef cattle for growth. Livestock Production Science, v.86, p.69-83, 2004. DOI: 10.1016/S0301-6226(03)00142-8.

MISZTAL, I.; TSURUTA, S.; LOURENÇO, D.; AGUILAR, I.; LEGARRA, A.; VITEZICA, Z. Manual for BLUPF90 family of programs. Athens: University of Georgia, 2014. 124p.

MOTA, R.R.; MARQUES, L.F.A.; LOPES, P.S.; SILVA, L.P. da; HIDALGO, A.M.; LEITE, C.D.S.; TORRES, R.A. Random regression models in the evaluation of the growth curve of Simbrasil beef cattle. Genetics and Molecular Research, v.12, p.528-536, 2013. DOI: 10.4238/2013.February.27.2.

NOBRE, P.R.C.; MISZTAL, I.; TSURUTA, S.; BERTRAND, J.K.; SILVA, L.O.C.; LOPES, P.S. Analyses of growth curves of Nellore cattle by multiple-trait and random regression models. Journal of Animal Science, v.81, p.918-926, 2003.

PEREIRA, R.J.; BIGNARDI, A.B.; EL FARO, L.; VERNEQUE, R.S.; VERCESI FILHO, A.E.; ALBUQUERQUE, L.G. Random regression models using Legendre polynomials or linear splines for test-day milk yield of dairy Gyr (Bos taurus) cattle. Journal of Dairy Science, v.96, p.565-574, 2013. DOI: 10.3168/ jds.2011-5051.

RAFTERY, A.E.; LEWIS, S.M. One long run with diagnostics: implementation strategies for Markov chain Monte Carlo. Statistical Science, v.7, p.493-497, 1992. DOI: 10.1214/ ss/1177011143.

REKAYA, R.; CARABAÑO, M.J.; TORO, M.A. Use of test day yields for the genetic evaluation of production traits in Holstein-Frisian cattle. Livestock Production Science, v.57, p.203-217, 1999. DOI: 10.1016/S0301-6226(98)00181-X.

SANTOS, G.C. de J.; LOPES, F.B.; MARQUES, E.G.; SILVA, M.C. da; CAVALCANTE, T.V.; FERREIRA, J.L. Tendência genética para pesos padronizados aos 205, 365 e 550 dias de idade de bovinos Nelore da região Norte do Brasil. Acta Scientiarum. Animal Sciences, v.34, p.97-101, 2012.

SCALEZ，D.C.B.; FRAGOMENI，B.O.; PASSAFARO, T.L.; PEREIRA, I.G.; TORAL, F.L.B. Polynomials to model the growth of young bulls in performance tests. Animal, v.8, p.370-378, 2014. DOI: $10.1017 / \mathrm{S} 1751731113002334$.

SMITH, B.J. Bayesian output analysis program (BOA) version 1.1 user's manual. 2005. 43p. Disponível em: <http://www. public-health.uiowa.edu/boa/boa.pdf>. Acesso em: 10 nov. 2013.

SOUZA, J.C.; SILVA, L.O.C.; GONDO, A.; FREITAS, J.A.; MALHADO, C.H.M.; FERRAZ FILHO, P.B.; SERENO, J.R.B.; WEABER, R.L.; LAMBERSON, W.R. Parâmetros e tendência genética de peso de bovinos criados a pasto no Brasil. Archivos de Zootecnia, v.60, p.457-465, 2011. DOI: 10.4321 / S0004-05922011000300035.

TORAL, F.L.B.; PEREIRA, J.C.C.; BERGMANN, J.A.G.; JOSAHKIAN, L.A. Parâmetros genéticos do peso desde o nascimento até 730 dias de idade na raça Indubrasil. Pesquisa Agropecuária Brasileira, v.49, p.595-603, 2014. DOI: 10.1590/ S0100-204X2014000800003.

VALENTE, B.D.; SILVA, M.A.; SILVA, L.O.C.; BERGMANN, J.A.G.; PEREIRA, J.C.C.; FRIDRICH, A.B.; FERREIRA, I.C.; CORRÊA, G.S.S. Estruturas de covariância de peso em função da idade de animais Nelore das regiões Sudeste e Centro-Oeste do Brasil. Arquivo Brasileiro de Medicina Veterinária e Zootecnia, v.60, p.389-400, 2008. DOI: 10.1590/S0102-09352008000200018.

Recebido em 24 de junho de 2015 e aprovado em 19 de novembro de 2015 